\title{
The Moon in the Landscape: Interpreting a Theme of Nineteenth Century Art
}

\section{Gary Wells}

\begin{abstract}
The image of the moon in the rural landscape is such a familiar and common theme in nineteenth century art that we should ask what made this theme so popular, widespread, and persistent. The similarity among these depictions borders on formula: a field or rustic farm, a broad horizon, a full moon rising or a thin crescent moon setting, perhaps a shepherd or field worker silhouetted against the twilight sky. But what made this image so appealing to nineteenth century artists and their audiences? This paper will examine the theme of the moon in the landscape, and will suggest that the persistence of the motif masks an evolving set of ideas about time, nature and change. From the personal visions of Samuel Palmer and Vincent van Gogh, to the contemplation of nature's sublimity in Caspar David Friedrich and Thomas Moran, the expressive range of the subject is significant. But a common thread emerges when these images are seen within the context of the nineteenth century's rapid industrialization, urbanization, and materialism. Rather than romantic invention or picturesque scenery, images of the moon in nineteenth century landscape art were used to explore a broad range of ideas about modernity, nature and humanity in an age of science and industry.
\end{abstract}

Of all the images that speak to the inspiration of astronomical phenomena and the sky, that of the moon in the landscape is the most familiar and iconic. For some, this is the definitive image of the sky (Figure 1). The picture of the nearby earth and the distant moon, of contrasting darkness and light, neatly sums up the entire experience of the sky. But after countless examples, it is an image that has passed from profundity to present day cliché. This was not always so, and as recently as the nineteenth century the image of the moon in the landscape still resonated with artists as the essential pictorial representation of a particular way of thinking about art, science, and nature.

I initially approached this topic as a survey of a motif during the apex of the landscape painting tradition in the nineteenth century. I wanted to present an overview of the moon in the landscape as emblematic of a certain emerging understanding of nature in the age of

Gary Wells, 'The Moon in the Landscape: Interpreting a Theme of Nineteenth Century Art', eds. Nicholas Campion and Rolf Sinclair, Culture and Cosmos, Vol. 16 nos. 1 and 2, 2012, pp. 373-384.

www.CultureAndCosmos.org 
374 The Moon in the Landscape: Interpreting a Theme of Nineteenth Century Art

science, inspired by the sentiment contained in John Constable's statement in 1836: 'Painting is a science and should be pursued as an inquiry into the laws of nature. Why, then, may not a landscape be considered as a branch of natural philosophy, of which pictures are but experiments?'

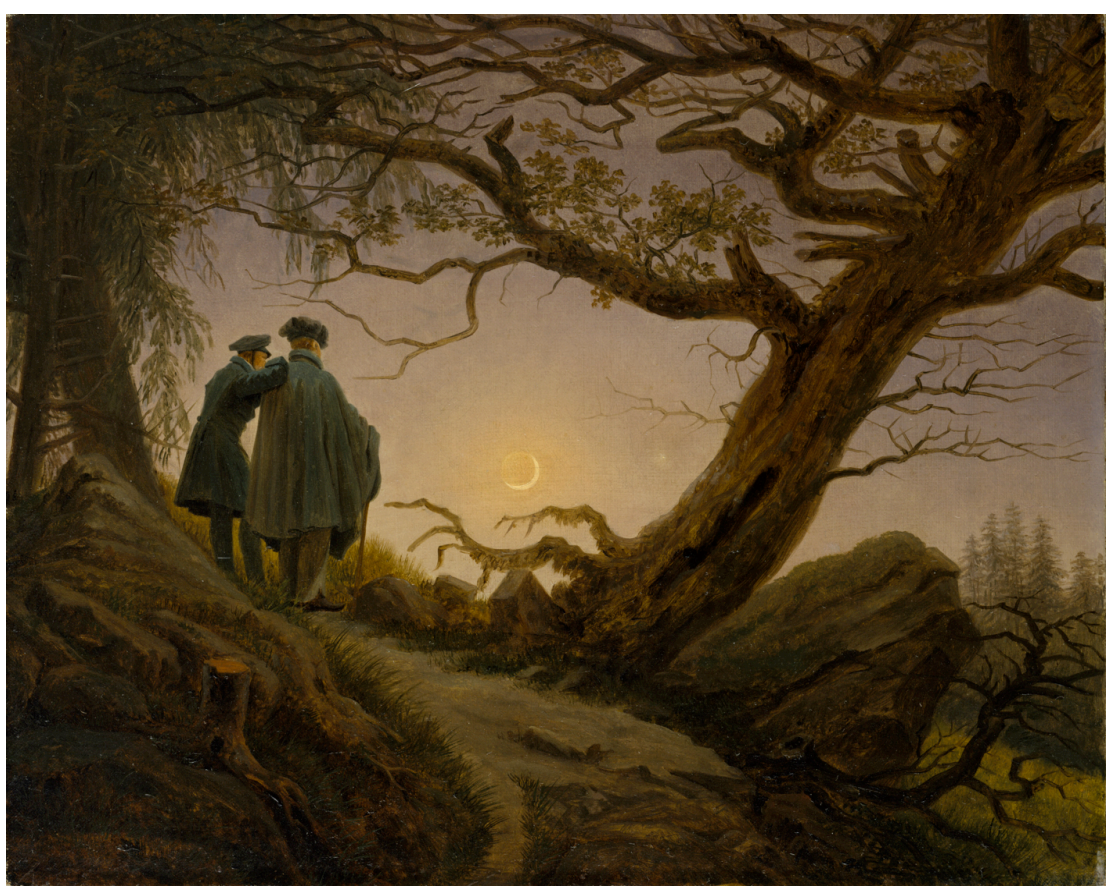

Figure 1. Caspar David Friedrich, Two Men Contemplating the Moon, c. 1830. Oil on canvas. The Metropolitan Museum of Art, New York, Wrightsman Fund, 2000 (2000.51).

At the intersection of art and astronomy, I even wanted to see a metaphor at work: the moon reflecting sunlight to illuminate the night seemed like an apt symbol of art reflecting the light of science to enlighten the dark corners of the human experience. During a century that had developed an implicit confidence in science to illuminate the mysterious, allegory and metaphor were still more important than raw reality. My motivating

${ }^{1}$ John Constable, John Constable's Discourses, ed. R.B. Beckett (Ipswich, Suffolk Records Society, 1970), p. 69.

Culture and Cosmos 
question was: what does this motif tell us about the two ways of regarding the sky, one artistic and the other scientific?

The scientific view of the image of the moon in the landscape may be found in such journals as Nature. Nature began to feature reviews of art exhibitions such as those at the Royal Academy, and general comments about the level of scientific knowledge reflected in the art of the time were central to those editorials. There is much interest specifically in landscape painting and in the details of geology, meteorology, and astronomy that appear in such works. Many of these columns on art were written by J. Norman Lockyer, founder and first editor of Nature, an astronomer with a special interest in spectroscopy, credited with the co-discovery of helium.

Lockyer's comments on landscape painting are chiefly concerned with the degree of scientific accuracy displayed by the artists. In the astronomical domain, he criticizes artists for their lack of apparent 'common sense' in presenting astronomically impossible scenes: crescent moons opposite the setting sun, full moons enlarged to gigantic proportions, misplaced constellations, and the like. 'It is, perhaps, in the case of reflection of light by the poor moon that the modern artist comes to the greatest grief...If an artist would amuse himself any evening with his children in imitating these conditions with a lamp and some oranges he would never make another mistake'. ${ }^{2}$ A grateful reader responded to Lockyer's helpful advice: 'Mr. Norman Lockyer...gives some valuable hints to artists, which, if carried out, will go a great way towards preventing our eyes being hurt by the lunar monstrosities we see at the Royal Academy and elsewhere'.

These comments, and others like them, are striking in their almost total lack of interest in the issues that motivate artists. Lockyer's belief that landscape paintings are either right or wrong, prone to 'mistakes', might strike some of us as incredibly presumptuous and remarkably ignorant of the history of art (the latter a fault that Lockyer readily acknowledged). It also has fallen to the wrong side of the later history of art-consider that the comments above were made in 1878 , just as the Impressionists in France were encountering their most severe criticism, and a little more than a decade before Vincent van Gogh would do everything wrong in creating the most iconic images of the night sky from the entire century. But the nineteenth century scientist as art critic

\footnotetext{
2 J. Norman Lockyer, 'Physical Science for Artists, II', Nature, Vol. 18 (May 16, 1878): p. 59.

${ }^{3}$ Robert J. Lecky, 'Letter to the Editor', Nature, Vol. 18 (May 30, 1878): p. 116.
} 
376 The Moon in the Landscape: Interpreting a Theme of Nineteenth Century Art

had a narrow view of art. Constable's ideas notwithstanding, most artists saw science as a means and not an end for art. The 'errors' noted by Lockyer were common, widespread, and persistent across the nineteenth century. Obviously, the intersection of art and science did not necessarily result in a trend toward greater visual or scientific accuracy.

Part of the work that I am doing to better understand this relationship of nineteenth century art and science led me to conduct a rather unscientific examination of a random sample of 178 landscape paintings by 104 artists done in Europe and North America between 1800 and 1900, all featuring the moon or moonlight in some aspect. The breakdown indicates that the full moon appears in nearly $60 \%$ of the works $(103$ of $178,57.87 \%)$, an early or late crescent in slightly fewer than $20 \%$ ( 35 of $178,19.66 \%$ ), a gibbous moon in 5.6\% (10 of 178), and over $16 \%$ are indeterminate or ambiguous (29 of 178, 16.29\%). Only one painting out of the group of 178 showed a distinct quarter moon (half the disk visible). The indeterminate paintings usually had the moon obscured behind clouds, or off the edge of the painting completely, where only a reflection of scattered moonlight was present in the image itself.

First, this perhaps simply confirms the obvious - artists liked to paint the full moon in the landscape. When they were not showing us the full moon, they were painting crescent new or old moons. This formula holds true, I suspect, not only for the nineteenth century, but also for the entire tradition of the moon as artistic motif. Second, the selectivity that artists exercised reflected aesthetic, rather than scientific, concerns, which we will return to in a moment.

In looking at writings on landscape painting in the nineteenth century outside the science journals, such as artists' handbooks, lectures, diaries and theoretical treatises, it becomes immediately clear that many, if not most, artists were familiar with the scientific, astronomical, reality of the night sky. This knowledge helped shape a general notion of the 'truth' in nature, and motivated artists to suggest that observation and knowledge were intimately connected. But it is also clear that these writings drew a line between the recording of observation and the production of art. While there are many artists who show what Lockyer would have called 'correct' skies (Frederic Church, for instance, comes close, in works like 'Cayambe', 1858, Museum of Fine Arts, Boston), it would difficult to say that this was their primary interest or the essential goal of their painting. Art, for much of the nineteenth century, was about bending nature to the creative will, rather than slavish subordination to observation alone.

Culture and Cosmos 
Further, the artwork of the era was inevitably seen in terms of meaning and content before representation. The nineteenth century's depictions of the sky spanned the entire representational spectrum from abstraction to realism. Even when they knew better, artists often chose to depict the sky in ways that contradict astronomical reality, if it allowed them to develop the expressive, emotional, symbolic, or narrative aspects of the scene. This last point is worth emphasizing, because it focuses our attention on the central aspect of nineteenth century art that is most often forgotten today: the nineteenth century artist and his audience were primarily iconographers. There was almost no representation that was not also symbolic, and there was certainly no general attitude of 'objective' representation even amongst professed 'realists'. Simply put, the image of the moon was always perceived as symbolic - there was no such thing as an objective image of the moon in fine art in the nineteenth century (Figure 2). And so the argument that scientific accuracy would not compromise artistic integrity, which Lockyer states repeatedly, bumps up against a complicated web of artistic intentions.

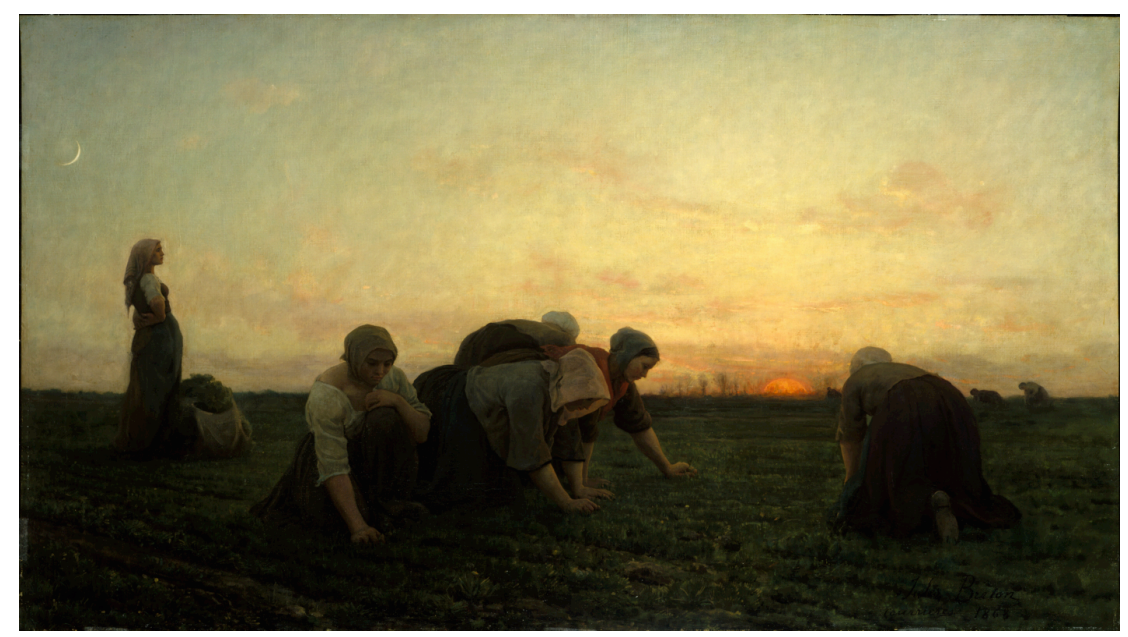

Figure 2. Jules Breton, French, The Weeders, 1868. Oil on canvas. The Metropolitan Museum of Art, Bequest of Collis P. Huntington, 1900 (25.110.66).

Artists saw no contradiction in professing, like Constable, that they approached nature truthfully, even scientifically, while subjecting the image of nature to the same abstractions, distortions, exaggerations, and outright inventions as any other subject. This is because the observation 
378 The Moon in the Landscape: Interpreting a Theme of Nineteenth Century Art

of reality is often the beginning, not the end, of the representation. This attitude was expressed in a passage from an 1887 artist's guide to landscape painting:

We are all of us visionary artists for one familiar object, the moon. We do not think of the heavy globe of rock with prodigious cloudless mountains, sun-heated to an intolerable temperature. This is the scientific conception that we keep in some odd corner of the brain for use when it may be wanted, as one keeps a scientific instrument in a drawer, but in ordinary times the moon means for us a crescent or a disc of silvery and sometimes golden splendour, the brightest thing that we are able to look upon in nature. Now to sever, in this way, the splendour of the moon from the idea of her reality, her substance, is exactly the artistic way of seeing. ${ }^{4}$

The scientific critique of nineteenth century landscape art underwent something of a revival with the publication of Albert Boime's analysis of Vincent van Gogh's 'Starry Night' in $1984 . .^{5}$ A cottage industry in comparative visualization infiltrated the study of astronomically inspired art of the nineteenth and twentieth centuries. While this scholarly trend has addressed questions of dating and of location in a handful of artworks, it has also dusted off a false assumption that representational fidelity is the basic criterion, or at least the intentional goal, of such images. Boime himself did not argue that the accuracy of Van Gogh's representation of the night sky was separate from the expressive and abstract qualities of the painting. But he did want to show that the sky is not 'made up' from the imagination, but instead extracted from observations made by the artist from nature itself. Perhaps this is persistent modern day defensiveness about Van Gogh's abstraction.

But the misapplication of this kind of analysis may reveal certain scientifically valid facts, or mistakes, in the paintings in question without fully comprehending the context, intentions, or reception of the works themselves. The conclusion that one might draw is that the primary aim of interpreting images of the sky in nineteenth century art is to find their

\footnotetext{
${ }^{4}$ Philip Gilbert Hamerton, Imagination in Landscape Painting (Boston: Roberts Brothers, 1887), p. 72.

5 Albert Boime, 'Van Gogh's Starry Night: A History of Matter and a Matter of History', Arts Magazine, Vol. LIX, no. 4 (December 1984): pp. 86-103.
} 
source in a specific experience, make the case for that source by appealing to a scientific verification of time and place, and conclude that the job has been done. But this fails to tell us about what the artists themselves were interested in expressing.

Consider, for instance, our earlier observation that well over half of the depictions in our sample show us the full moon. Of course, the full moon appears only one day out of twenty-eight in reality, and even if we expand the window a day or so on either side, the observation of the full moon corresponds to roughly one-tenth of the lunar cycle. Of course, the full moon is the most visually dramatic aspect of that cycle, and has the added advantage of providing the maximum illumination in the landscape below. But the obvious selectivity with which artists show us landscapes with full moons should indicate something about the choices that they make in creating a painting.

Nineteenth century artists who depicted the moon in the landscape may have recognized the scientific rationale for phenomena in nature, but many were also painting the received wisdom of convention despite their frequent direct contact with nature outside the studio. They needed to solve certain practical problems of representation in their works first. An artist aspiring to a modicum of representational fidelity would note that the full moon is prominent not only because it is bright, but also because it is situated in a field of view that is easily observed and conveniently timed (Figure 3 ).

The field of vision in the traditional landscape format of nineteenth century painting limited the amount of sky that could be depicted. The ratio of height to width of canvases used for landscape and seascape paintings was not precisely standardized, but nineteenth century suppliers tended to sell pre-stretched canvases with a roughly 3 to 2 ratio of width to height (seascapes were wider in proportion to their height, closer to 2 to 1 ). The horizontal orientation of most landscape paintings usually covered a 60 degree-wide field of view, which limited the vertical angle of view to less than $25^{\circ}$ above the horizon, even when the horizon was depressed below the midline of the painting. While it is possible to create a landscape painting with a larger vertical field of view-for instance, by turning the canvas into a vertical orientation - this is rare and, to many viewers, unnatural. 
380 The Moon in the Landscape: Interpreting a Theme of Nineteenth Century Art

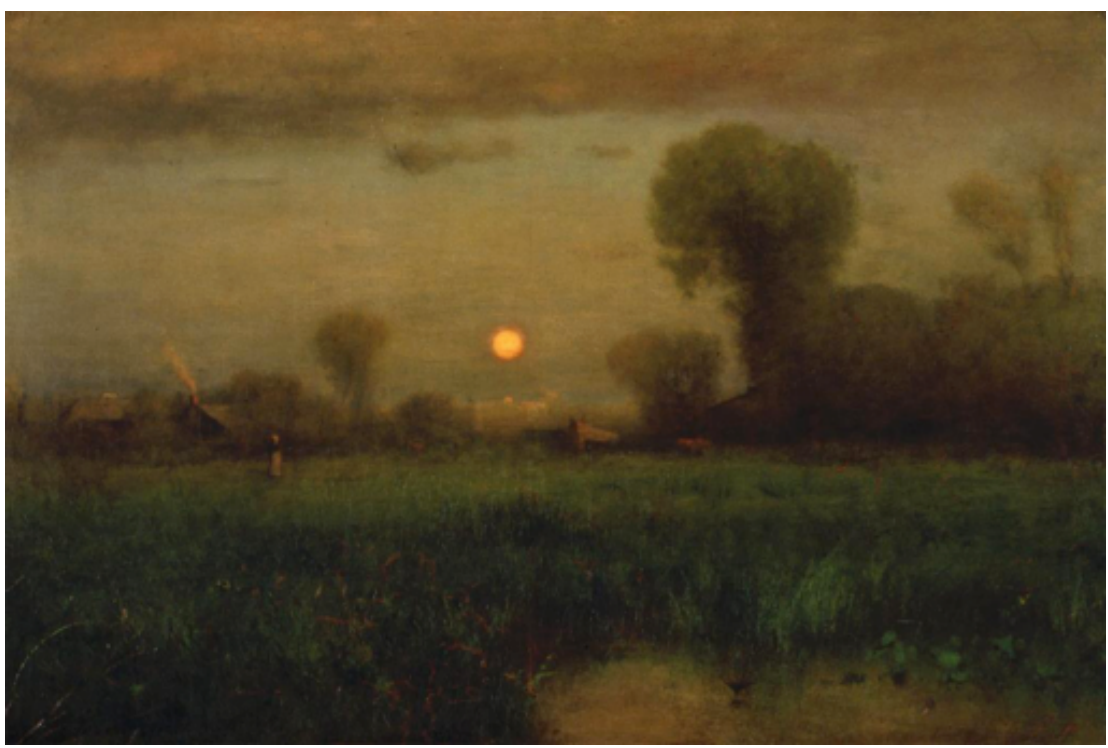

Figure 3. Inness, George, Harvest Moon, 1891. Oil on canvas. Corcoran Gallery of Art, Washington, D. C. Bequest of Mabel Stevens Smithers, The Frances Sydney Smithers Memorial.

Now this matters because the limited slice of the sky that artists had to work with determined the kind of phenomena that they could depict. The full span of celestial phenomena must be compressed into a relatively small slice of the sky near the horizon. It is only when the moon is relatively low to the horizon that it becomes visually important to most artists. Thus, the phenomena of the sky that fall within this narrow range, comprising less than one-sixth of the horizon-to-horizon sky dome, are the most likely to be depicted by the artist and that appear 'natural' to the observer. A full moon, hanging near the horizon opposite the setting sun, was visually prominent. So, too, was the thin crescent of the new moon hovering above the western horizon immediately after sunset.

Gibbous phases were timed awkwardly for most artists to take special notice, and were aesthetically less interesting. It was difficult to place the gibbous phases into the restricted visual space of the conventional landscape painting without a special context (see for example, Jean-François Millet's 'The Sheep Pen in Moonlight', 1872, Musée d'Orsay, Paris). And while there are many instances of indirect representation of the moon outside the frame of the painting-for 
instance, moonlight reflecting upon water-the actual image of the moon itself was far more common.

But what did it mean? As a subject, the image of the moon in the landscape was a special case for artists, a theme used sparingly and specifically. The moon was most often used as an oppositional elementnight against day, dark against light, rising against setting. While its traditional symbolic associations with time, femininity, and cyclical change were still relevant to the nineteenth century, the moon was also associated with images of the passing of cultural or social epochs. Thomas Cole's end of empire (for example, 'Course of Empire: Desolation', c. 1848, New York Historical Society, New York), JeanFrançois Millet's peasants, and Jasper Cropsey's view of an ancient Greek temple ('Evening in Paestum', 1856, Vassar College Art Gallery, Poughkeepsie, NY) all use the moon as a symbolic marker of the passing of a way of life, of a society, or of an era. Contrarily, the moon could also mean the beginning of a new era, either as the thin new moon crescent that appears as the sun sets, or as the full moon that rises opposite the setting sun. Both situations play off the setting sun as a traditional symbol of endings and conclusions, with the new moon hinting at the beginning of a new cycle, and the full moon as the rising opposite of the setting sun, the substitute light that replaces the waning age.

But most images of the moon in the landscape are far less specific. Handbooks of nineteenth century painting explain that moonlight is evocative, mysterious, and expressive: 'Moonlight possesses great charms for all lovers of nature. Objects seen by it seem separated from the more common light and influence of every-day life, and subject to different laws, as if they belonged to another world'. ${ }^{6}$ This otherworldliness could evoke dreams or nightmares, as artists like Albert Pinkham Ryder would explore ('The Temple of the Mind', c. 1885, Albright-Knox Art Gallery, Buffalo, NY). Or they could simply be the Romantic vision of a spiritual or emotional space, like the calm vistas of Caspar David Friedrich or the dramatic stage of Ralph Blakelock (for example, Moonlight, c. 1885, Brooklyn Museum of Art). Moonlight shone on fires, shipwrecks and disasters in early nineteenth century Romantic paintings, but then was tempered by second generation Romantics into an element of exoticism, especially suited to a symbolic role in Orientalist scenes of the desert Middle East. But for most

\footnotetext{
${ }^{6}$ George Barnard, The Theory and Practice of Landscape Painting in WaterColours (London: Routledge, Warne, and Routledge, 1859), p. 240.
}

Culture and Cosmos 
382 The Moon in the Landscape: Interpreting a Theme of Nineteenth Century Art

landscape artists, the moon was simply an evocative part of the twilight, that intermediary time between day and night. The theme of the nocturne grew in popularity as the century progressed, replacing earlier Romantic and Realist views, so that by the 1880s and 1890s it was the most familiar context for the moon in the landscape. American painter George Inness, for example, virtually made a career out of hazy, evocative scenes of orange moons, suggestive shadows, and mysterious women.

But I think that the moon in the landscape was much more to the artists and audiences of the nineteenth century. Theirs was a time of wide contrasts between past and present, reason and emotion, optimism and despair. Art was a way of making sense of the world, much as science struggled to do the same, and through the century art and science moved in parallel. John Constable said, 'We see nothing truly till we understand it'?

The nineteenth century believed that the world and the universe was knowable, but born of this knowledge was a melancholy materialism that replaced earlier sentiments of the divine. The moon was a suitably malleable and ambiguous symbol to express these contradictions and doubts. Van Gogh's moons seek to hold onto the spiritual in nature, but as the century closed, a different vision of the moon came to dominate the public imagination. Photography and increasingly powerful telescopes brought the moon closer, pushing the landscape out of the picture and focusing our attention upon the moon as a place. Engineer and astronomer James Nasmyth imagined the distant world not in the landscape, but itself as a landscape:

While earnestly studying the details of the moon's surface it was a source of great additional interest to me to endeavor to realize in the mind's eye the possible landscape effect of their marvelous elevations and depressions. Here my artistic faculty came into operation. I endeavored to illustrate the landscape scenery of the moon in like manner as we illustrate the landscape scenery of the earth. ${ }^{8}$

${ }^{7}$ C. R. Leslie, Memoirs of the Life of John Constable, Esq. R.A., Composed Chiefly of His Letters (London: Longman, Brown, Green, and Longmans, 1845), p. 350 .

${ }^{8}$ James Nasymth, James Nasmyth, Engineer: An Autobiography (New York: Harper \& Brothers, 1883), p. 336. 
Imaginative invention turned from recording the direct observations of the sky to a speculative visualization, and in Camille Flammarion's Astronomie Populaire of 1880, the image of the earth hanging in the lunar sky inverts the common trope of the moon in the landscape (Figure 4). ${ }^{9}$

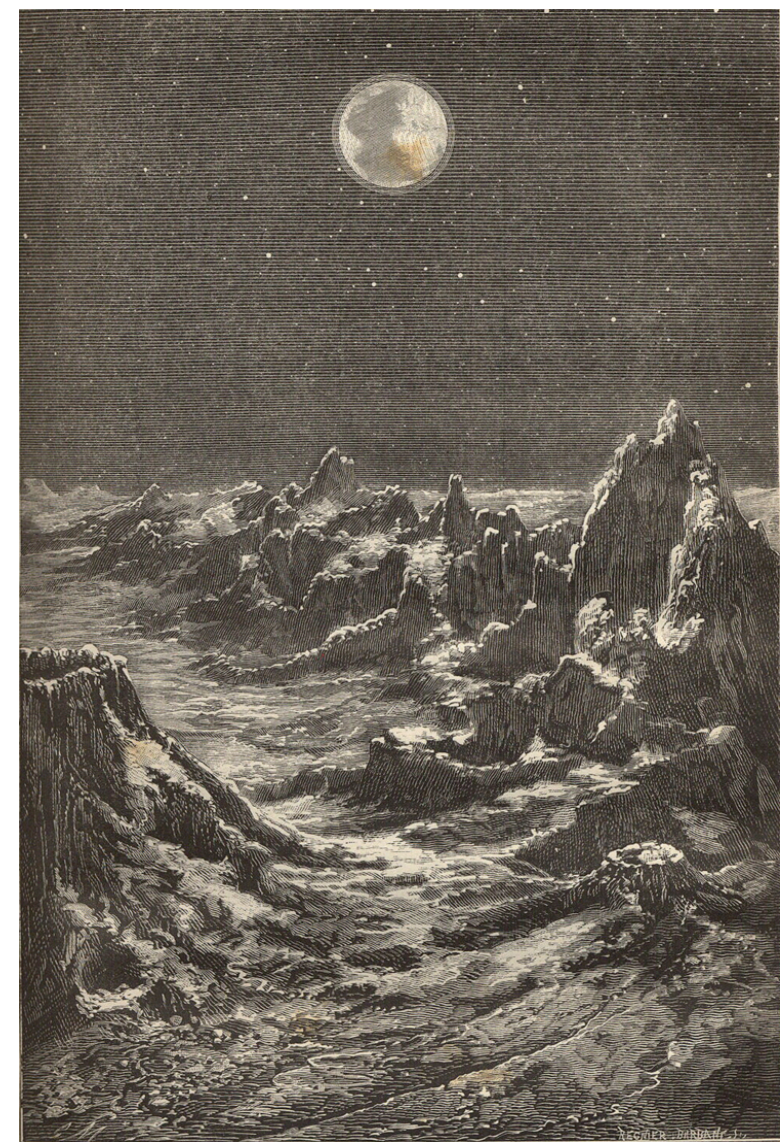

Figure 4. Camille Flammarion (A. Regnier, artist; Charles Barbant, engraver), Full Earth, seen from the Moon, plate 26 from Astronomie populaire, Paris: 1880

9 Camille Flammarion, Astronomie Populaire (Paris: C. Marpon et E. Flammarion, 1880), plate 26. 
384 The Moon in the Landscape: Interpreting a Theme of Nineteenth Century Art

The alpine mountains and dramatic chiaroscuro are borrowed directly from the nineteenth century's landscape traditions. Nasmyth and Flammarion both realized that science could harness artistic vision, could play upon both the visual and the iconographical familiarity of the moon in the landscape, to visualize what human observers had never witnessed. Their perspective of the lunar landscape as the inverse of the earthly landscape was to foretell the most striking image of the century yet to come, not of the moon in the earthly landscape, but of the earth seen by the Apollo astronauts rising over the lunar landscape. 